\title{
A Prospective Study of Social Competence in Survivors of Pediatric Brain Tumors
}

\author{
May Albee ${ }^{1}$, Santiago Allende ${ }^{2}$, Victoria Cosgrove ${ }^{2}$, and Matthew Hocking ${ }^{1}$ \\ ${ }^{1}$ The Children's Hospital of Philadelphia \\ ${ }^{2}$ Stanford University School of Medicine
}

November 29, 2021

\begin{abstract}
BACKGROUND/OBJECTIVES: Survivors of pediatric brain tumors (BT) are at increased risk for difficulties with social competence, including poor social information processing (SIP) and peer relationships. Due to improved survival rates among BT, there is a need to better understand these challenges and if they are specific to BT versus other survivors of childhood cancer. METHODS: $51 \mathrm{BT}$ and 34 survivors of pediatric solid tumors (ST) completed evaluations of SIP and peer relationship quality within 6 months of completing treatment and at one year follow-up. Caregivers also completed a measure of social skills. Linear mixed models evaluated (1) differences between BT and ST on SIP and social skills and (2) how indices of SIP were associated with peer relationships over time for ST and BT. RESULTS: BT did not differ from ST on indices of SIP or social skills over time. There was a three-way interaction between measures of SIP, group, and time to predict peer relationships. ST showed a positive association between baseline social skills and theory of mind and peer relationships over time, whereas BT showed an inverse association between baseline social skills and theory of mind and peer relationships over time. CONCLUSION: Baseline SIP and social skills affected the trajectory of BT peer relationships. BT social functioning should be monitored regularly after the completion of treatment to determine if and when intervention services would be beneficial.
\end{abstract}

A Prospective Study of Social Competence in Survivors of Pediatric Brain and Solid Tumors

May Albee $\mathrm{BS}^{1}$, Santiago Allende PsyD ${ }^{2}$, Victoria Cosgrove $\mathrm{PhD}^{2}$, \& Matthew C. Hocking $\mathrm{PhD}^{1,3}$

${ }^{1}$ The Children's Hospital of Philadelphia, Philadelphia, PA

${ }^{2}$ Stanford University School of Medicine, Palo Alto, CA

${ }^{3}$ Perelman School of Medicine at the University of Pennsylvania, Philadelphia, PA

Corresponding Author:

Matthew C. Hocking

The Leonard \& Madalyn Abramson Research Center

3615 Civic Center Blvd, Office 1427B

Philadelphia PA 19104

(267) 426-5561

hockingm@chop.edu

Abstract Word Count: 228

Manuscript Word Count: 3,106 
4 Tables, 2 Figures

Key Words: Brain Tumor, Solid Tumor, Social Information Processing

Running Title: Social Competence in Survivors of Pediatric Cancer

Abbreviation Key

\begin{tabular}{ll}
\hline BT & Survivors of Pediatric Brain Tumors \\
\hline CNS & Central Nervous System \\
ST & Survivors of non-CNS Solid Tumors \\
SIP & Social Information Processing \\
ASD & Autism Spectrum Disorder \\
DANVA-2 & Diagnostic Analysis of Nonverbal Accuracy Child Faces 2 \\
SSiS & Social Skills Improvement System \\
PROMIS & Patient Reported Outcomes Measurement Information System \\
PNORTI & Pediatric Neuro Oncology Rating of Treatment Intensity \\
NPS & Neurological Predictors Scale \\
\hline
\end{tabular}

\section{ABSTRACT}

BACKGROUND/OBJECTIVES: Survivors of pediatric brain tumors (BT) are at increased risk for difficulties with social competence, including poor social information processing (SIP) and peer relationships. Due to improved survival rates among BT, there is a need to better understand these challenges and if they are specific to BT versus other survivors of childhood cancer.

METHODS: 51 BT and 34 survivors of pediatric solid tumors (ST) completed evaluations of SIP and peer relationship quality within 6 months of completing treatment and at one year follow-up. Caregivers also completed a measure of social skills. Linear mixed models evaluated (1) differences between BT and ST on SIP and social skills and (2) how indices of SIP were associated with peer relationships over time for ST and BT.

RESULTS: BT did not differ from ST on indices of SIP or social skills over time. There was a three-way interaction between measures of SIP, group, and time to predict peer relationships. ST showed a positive association between baseline social skills and theory of mind and peer relationships over time, whereas BT showed an inverse association between baseline social skills and theory of mind and peer relationships over time.

CONCLUSION: Baseline SIP and social skills affected the trajectory of BT peer relationships. BT social functioning should be monitored regularly after the completion of treatment to determine if and when intervention services would be beneficial.

\section{INTRODUCTION:}

Survivors of pediatric brain tumors (BT) are increasing in number due to advances in treatment over the past 20 years [1]. The original focus on survival for this population has shifted over time to encompass the cognitive, physical, and social ramifications of disease and treatments on individuals from diagnosis through the course of their lives. With a more holistic understanding of the impact of cancer treatment on the developing central nervous system (CNS), more recent research has shown that BT are less likely than typically developing peers to go to college, live independently, have close friends, and marry [2]. In the short-term, BT also struggle socially and are less likely to establish friendships compared to survivors of non-CNS tumors (ST) and are more socially isolated than their peers [3, 4]. The majority of studies of social functioning in BT has been cross sectional in nature, and longitudinal research is needed to better understand the trajectory and predictors of social functioning in BT over time. 
A model of social competence in children with insults to the brain [5] integrates risk and resilience factors related to CNS insults, the family environment, and individual characteristics and offers direction for research. Insults to the developing brain may impair cognitive and executive functioning which can in turn lead to poor social skills and, subsequently, poor social adjustment, including peer relationships [6]. Social skills are defined as skills required to interact appropriately across a variety of social contexts [7]. Developmentally inappropriate social skills may cause individuals to react inappropriately to social situations, in turn leading to peer isolation and rejection. This model of social competence has been applied to the BT population [8] as they experience social late effects as a result of CNS insult.

Social Information Processing (SIP) is emphasized as a key determinant of social interaction and social adjustment. Two important elements of SIP include properly identifying facial expressions and correctly interpreting others' motivations, also known as theory of mind [5]. These are common struggles in youth with Autism Spectrum Disorder (ASD) [9] which contribute to difficulties with social interactions [10]. If an individual is unable to correctly identify the emotion of another, it becomes far more difficult to react in a socially appropriate manner which can lead to social isolation and peer rejection. The inability to understand when an individual's comments are sarcastic or ironic can cause someone to misinterpret the speakers' intent and hinder appropriate social responses. While there is a large body of research examining these domains of social deficits in youth with ASD, research in BT is in its inception [8]. Preliminary studies have found that BT perform significantly worse than youth with juvenile rheumatoid arthritis in identifying adult facial expressions [11] and that BT with high levels of social impairment were worse at identifying facial expressions compared to typically developing youth and youth with ASD [12]. Longitudinal research is needed to better understand the social competence of BT over time in order to inform targeted interventions.

The goal of this longitudinal study was to evaluate important facets of social competence among ST and BT over time and to identify predictors of peer relationships. We hypothesized that (a) BT will have worse SIP and social skills over time compared to ST and that (b) worse SIP and social skills would predict worse peer relationship quality.

\section{METHODS:}

\section{Participants :}

Participants were youth ages 7-14 who had completed tumor-directed treatment for either a non-central nervous system (CNS) solid tumor $(\mathrm{n}=34)$ or brain tumor $(\mathrm{n}=51)$ within the six months prior to enrollment. Survivors of pediatric non-CNS solid tumors (ST) were chosen as the comparison group because they undergo similar treatments and comparable changes to their daily life during treatment, yet treatment does not directly affect the brain and they do not tend to show difficulties with SIP in survivorship. Exclusion criteria included: (1) a multi-systemic genetic condition affecting cognitive functioning (e.g., Neurofibromatosis Type 1, Trisomy 21); or (2) a cognitive or developmental delay prior to tumor diagnosis. Survivors of non-CNS solid tumors who completed treatments affecting the CNS (e.g., total body irradiation) were excluded as well. Caregiver inclusion criteria consisted of living with the participant at least $50 \%$ of the time, being fluent in English, and having actively participated in the participants' cancer treatment.

127 families were approached for this study ( $\mathrm{n}=72 \mathrm{BT}, \mathrm{n}=55 \mathrm{ST})$ and $83(65.3 \%, \mathrm{n}=49 \mathrm{BT}, \mathrm{n}=34$ ST) agreed to participate. Reasons for declining participation included lack of interest $(n=22)$, being too busy to participate $(n=7)$, and passive refusal $(n=15)$. Two BT were unable to complete study procedures upon enrollment due to language barriers resulting in a total sample of 81 . There were no differences in demographic or medical characteristics between consenting and non-consenting individuals.

\section{Procedures :}

The current data are from a longitudinal study of social competence in survivors of pediatric brain and nonCNS solid tumors conducted at a large, urban pediatric medical center. All procedures were reviewed and approved by the Institutional Review Board and written informed consent and child assent were obtained upon enrollment. Potentially eligible patients were identified through cooperation with medical teams and 
tumor registry records. All potential participants were contacted by study staff via letter, phone, and/or during follow-up visits. This paper encompasses data from the baseline study visits and the first follow-up, which occurred approximately 12 months after the baseline visit. Participants and their primary caregiver were asked to complete questionnaires (see below) during study visits. Relevant information was collected via chart review. Participants received a brief feedback letter and a $\$ 20$ gift card as compensation after each study visit.

\section{Measures:}

\section{Social Information Processing}

The Diagnostic Analysis of Nonverbal Accuracy Child Faces 2 (DANVA-2) [13] assesses the ability of individuals to properly identify the facial expressions of children across four emotions (happiness, sadness, anger, and fear) and two levels of emotional intensity (high and low). Participants viewed 24 faces and were asked to select the emotion that they believed was being depicted. The DANVA-2 has established validity and has been shown to differentiate clinical groups [14].

The Literal Truth, Ironic Criticism, and Empathic Praise Task (Irony \& Empathy) [15] presents six situations in picture form along with a recorded narration of the situation (raking a pile of leaves, erasing a blackboard, making a cake). The narration has neutral, ironic, or empathic intonation and participants must properly identify the intention of the narration based on the image and recording. This measure is frequently used to identify SIP deficits in youth with traumatic brain injury [16].

\section{Social Skills}

The Social Skills Improvement System (SSiS) [17] is a widely-used parent reported measure of social skills, academic competence, and problem behaviors. Caregivers rate statements on social skills and problem behaviors on a scale from 0-3 (0 being never, 3 being almost always) over the past two months (i.e., expresses feelings when wronged; starts conversations with peers; gets distracted easily). A standard score for social skills is calculated where higher scores indicate better social skills.

Social Adjustment

The Patient-Reported Outcomes Measurement Information System (PROMIS) Pediatric Peer RelationshipsShort Form (PRS-SF) is a validated self and parent report measure of peer relationships [18]. Participants rate eight statements regarding peer relationships on a $0-4$ scale ( 0 being never, 4 being almost always) over the past seven days (i.e., I felt accepted by other kids my age, I was able to count on my friends). Appropriate items are reverse coded to produce a total $\mathrm{T}$ score, where higher scores indicate better peer relationships. The self-report PRS-SF has demonstrated significant correlations with peer-reported metrics of social acceptance [18].

\section{Data Analysis Plan}

Linear mixed models were used to evaluate whether (a) BT would have worse SIP and social skills over time compared to ST and (b) whether social skills and accuracy in recognizing facial expressions and others' social intentions were positively associated with peer relationship quality. For longitudinal designs with two time points and data missing at random, maximum likelihood estimation used in mixed models produces less biased estimates than least squares approaches [19]. Thus, we used a random intercept model with restricted maximum likelihood estimation to test our hypotheses. All continuous predictors were grand-mean centered to aid the interpretation of coefficients.

In the model for hypothesis (a), DANVA-2, Irony \& Empathy, and SSiS were entered as outcome variables in three separate models, while time, group and their interaction were entered as predictors. Three models were run for hypothesis (b) that differed only in terms of the specific predictor variable tested. The three predictors included baseline values of SSiS, DANVA-2, and Irony \& Empathy. For example, for the model evaluating the influence of baseline social skills, PROMIS Pediatric Peer Relationships was the outcome variable, while time, baseline SSiS, group and a 3-way interaction between them were entered as predictors. 
We also conducted two post hoc analyses in the BT group, where we evaluated the moderating effect of baseline time-since-diagnosis on Irony \& Empathy and social skills. Examination of diagnostic plots at levels 1 and 2 showed that models met assumptions of normality, linearity, independence and constant variance. The nlme package from the R Statistical Computing Language was used to run the linear mixed models and the sjPlot $\mathrm{R}$ package was used to produce tables and graphs of simple slopes.

\section{RESULTS}

\section{Descriptive and Preliminary Analyses}

Baseline visits were completed within six months of completing tumor-directed treatment $(M=3.39$ months, $\mathrm{SD}=1.78$ months, range $=2$ weeks- 6.9 months). Time 2 visits occurred an average of 13.1 months after baseline $(\mathrm{SD}=1.72$ months, range $=10.3-17.7$ months $)$.

Table 1 summarizes the sample's demographic characteristics at baseline. The majority of BT participants were male $(74.5 \%)$, and the majority of ST participants were female $(61.8 \%)$. A majority of both BT and ST were white $(74.5 \%$ and $61.8 \%$ respectively) and non-Hispanic ( $82.4 \%$ and $73.5 \%$ respectively). The most common treatment modality was surgical resection only for both BT and ST (33.3\% and 35.3\%, respectively). Other treatment combinations are provided in Table 1 . There were no differences in age, sex, or ethnicity for participants who only completed the baseline evaluation versus those who completed both baseline and follow-up. There was a significant difference in the ethnicity of BT participants who completed baseline only versus baseline and follow-up, however this difference is likely explained by relapse status. Three of the four BT participants who identified as Asian relapsed or passed away between

Indices of SIP and self-reported peer relationships did not change over time for either BT or ST. Self-reported peer relationships was in the average range overall, however $32.6 \%$ of BT had a score one standard deviation below average versus $15.6 \%$ of ST.

Hypothesis (a)

As assessed by DANVA-2 and Irony \& Empathy, BT and ST did not differ in SIP over time $(b=-0.00, s e=$ $0.01, t=-0.40 p=.694)$ and $(b=-0.01, s e=0.06, t=-0.13 p=.896)$, respectively (Table 2). Similarly, the model for SSiS failed to show longitudinal differences between BT and ST $(b=1.25$, se $=2.03, t=$ $0.62, p=.542)$

\section{Hypothesis (b)}

The 3-way interaction between time, group and social skills was significant (Table 3). In the ST group, there was a positive association between baseline social skills and peer relationships over time (Fig. 1). However, in the BT group, participants with greater social skills at baseline experienced a decrease in peer relationship quality over time, while those with lower social skills at baseline experienced an increase in peer relationship quality over time (Fig. 1).

The 3-way interaction between time, group and Irony \& Empathy was significant (Table 4). In the solid tumor group, there was a positive association between baseline Irony \& Empathy and peer relationships over time (Fig. 2). However, in the BT group, participants with greater baseline Irony \& Empathy scores showed evidence of a decrease in peer relationship quality over time, while those with lower baseline Irony \& Empathy showed evidence of an increase in peer relationship quality over time (Fig. 2).

The 3-way interaction between time, group, and DANVA-2 was not significant.

\section{Post Hoc Analyses}

Post hoc analyses were conducted for the BT group to help contextualize the unexpected, crossed interaction between baseline Irony \& Empathy and social skills and peer relationships over time. We examined whether the crossed interactions varied as a function of time-since-diagnosis, the intensity of treatment using the Pediatric Neuro-Oncology Rating of Treatment Intensity (PNORTI) [20] and treatment risk factors using the Neurological Predictor Scale (NPS) [21]. Results showed that the 3-way interaction between time, Irony 
\& Empathy and time-since-diagnosis was significant $(b=-0.04$, se $=0.01, t=-2.76, p=.011)$, with simple slopes demonstrating an increase in the crossed interaction between time and Irony \& Empathy with an increase in time-since-diagnosis. Results failed to demonstrate a significant 3-way interaction between time, social skills, and time-since-diagnosis $(b=-0.00$, se $=0.00, t=-0.91, p=.375)$. Results also failed to demonstrate moderating effects of treatment intensity or risk factors $(b=0.33$, se $=0.17, t=2.00, p=$ $.058)$ and $(b=0.09$, se $=0.06, t=1.46, p=0.159)$, respectively.

\section{DISCUSSION}

The goal of this longitudinal study was to evaluate components of social competence, including social information processing, social skills, and peer relationship quality, over time in survivors of pediatric brain tumors and non-CNS solid tumors who recently completed treatment. Due to improvements in survival rates among BT, there is now an emphasis on understanding their social functioning to better inform what supports may be needed throughout their lifetime. This is one of the first longitudinal studies to focus on SIP and peer relationships in BT and highlights the complexities in how SIP and social skills may relate to peer relationships in BT over time in comparison to ST. Among ST, we found positive associations between baseline measures of social skills and theory of mind and peer relationships over time. Conversely, BT showed an inverse association between baseline social skills and theory of mind and peer relationships over time. Those with better social skills and SIP at baseline had a decline in peer relationships, while those with worse social skills and SIP at baseline had an improvement in peer relationships over time. These findings add to a growing area of research evaluating the importance of social skills in BT $[5,15]$.

Our hypothesis that BT would have worse social skills and SIP compared to ST over time was not supported by the findings. There were no differences in social skills and SIP over time between these two groups and, notably, these variables did not change over time for either group. This could be because study assessments occurred within the first 18 months after treatment completion and may be too early to capture the neurodevelopmental late effects that arise in BT. A previous study found that BT who were farther out from end of treatment (3-9 years) showed significantly poorer face processing skills compared to youth with Juvenile Rheumatoid Arthritis [14]. Future longitudinal studies should follow BT for longer periods of time following treatment completion to identify when impairments in social skills and SIP develop.

Across the study, both BT and ST self-reported peer relationship scores were in the average range. However, the trajectory of self-reported peer relationship scores over time was affected by both group membership and baseline theory of mind and social skills. When comparing BT and ST on peer relationships over time, ST showed relatively stable peer relationships over time regardless of baseline theory of mind or social skills. However, for BT, social skills and theory of mind appeared to be more relevant to peer relationships over time in unanticipated ways. Face processing was not predictive of peer relationships in BT, which differs from previous cross-sectional studies which found associations poor face processing and social functioning [12, 22]. BT with significantly lower baseline social skills and theory of mind had improvements in peer relationships over time whereas BT with significantly higher baseline social skills and theory of mind had declines in peer relationships over time. Previous literature has noted a similar inverse association in processing speed abilities in survivors of pediatric brain tumors who received radiation therapy with those scoring the highest having the most marked decline in processing speed over time [23]. Moreover, exploratory post-hoc analyses of the present study suggested that this inverse association is most prominent in BT who were furthest from diagnosis which was seen in the previously mentioned study as well. Other factors, such as treatment modalities (e.g., radiation therapy) and neurological risk, did not affect this interaction in our analyses. Given that participants were enrolled in this study within 6 months of completing tumor-directed therapy, further time since diagnosis may be a proxy for more complex, lengthy treatments and, therefore, potentially having more sequelae.

Additionally, prior research suggests that BT may overestimate their social status among peers [24]. In the current study, participants rated their own levels of social acceptance by peers using the PROMIS measure. A potential explanation for these findings is that those with better baseline theory of mind may have better self-awareness of their status among peers and more accurately noted their decline in peer acceptance over 
time secondary to potential sequelae. Conversely, those with poorer baseline theory of mind may overestimate their peer acceptance over time due to difficulty interpreting social cues. Studies employing peer-reported data would be needed to elucidate these findings.

This study has several strengths. Data on the social competence of survivors of pediatric brain and solid tumors upon treatment completion is relatively limited. Additionally, the longitudinal nature of this study allows for evaluation of SIP and social acceptance over time to inform the provision of support services. Lastly, this study utilized a multi-informant method to evaluate variables of interest in survivors. Despite these strengths, these data should be viewed in the context of certain limitations. First, participant attrition led to smaller sample sizes at time point 2. Retention primarily was affected by participant relapses and deaths. Second, this study is not racially and ethnically diverse and findings may not generalize to the population writ large.

This study contributes to the growing body of research on the social competence of BT [14, 25] and offers numerous directions for future research and clinical efforts. Due to the variability in perceived social acceptance across time points, BT social functioning should be monitored regularly after the completion of treatment to determine if and when intervention services would be beneficial. Future research studies could expand on this work by following survivors for multiple years post-treatment completion and obtaining peer reports of survivor social acceptance.

\section{CoI STATEMENT}

The authors have no conflicts of interest to report

\section{ACKNOWLEDGEMENTS}

This research was supported by the National Cancer Institute of the National Institutes of Health (K07CA178100). The content is solely the responsibility of the authors and does not necessarily represent the official views of the National Institutes of Health. The authors thank all the participants who provided their time for this study.

\section{REFERENCES}

1. Noone, A., et al., SEER cancer statistics review, 1975-2015.Bethesda, MD: National Cancer Institute, 2018. 4 .

2. Zebrack, B.J., et al., Psychological outcomes in long-term survivors of childhood brain cancer: a report from the childhood cancer survivor study. Journal of Clinical Oncology, 2004. 22 (6): p. 999-1006.

3. Vannatta, K., et al., A Controlled Study of Peer Relationships of Children Surviving Brain Tumors: Teacher, Peer, and Self Ratings.Journal of Pediatric Psychology, 1998. 23 (5): p. 279-287.

4. Hocking, M.C., et al., Friendships in Pediatric Brain Tumor Survivors and Non-Central Nervous System Tumor Survivors. Journal of Pediatric Psychology, 2020. 45 (2): p. 194-202.

5. Yeates, K.O., et al., Social outcomes in childhood brain disorder: a heuristic integration of social neuroscience and developmental psychology. Psychological bulletin, 2007.133 (3): p. 535.

6. Yeates, K.O., et al., Social Outcomes in Childhood Brain Disorder: A Heuristic Integration of Social Neuroscience and Developmental Psychology. Psychological Bulletin, 2007.133 (3): p. 535-556.

7. Rubin, K.H. and L. Rose-Krasnor, Interpersonal problem solving and social competence in children, in Handbook of social development . 1992, Springer. p. 283-323.

8. Hocking, M.C., et al., Social competence in pediatric brain tumor survivors: Application of a model from social neuroscience and developmental psychology. Pediatric blood \& cancer, 2015.62 (3): p. 375-384.

9. Wolf, J.M., et al., Specific impairment of face-processing abilities in children with autism spectrum disorder using the Let's Face It! skills battery. Autism Research, 2008. 1 (6): p. 329-340. 
10. Mazza, M., et al., The role of theory of mind on social information processing in children with autism spectrum disorders: A mediation analysis. Journal of autism and developmental disorders, 2017. 47 (5): p. 1369-1379.

11. Bonner, M.J., et al., Social Functioning and Facial Expression Recognition in Survivors of Pediatric Brain Tumors*. Journal of Pediatric Psychology, 2008. 33 (10): p. 1142-1152.

12. Hocking, M.C., et al., Face Processing and Social Functioning in Pediatric Brain Tumor Survivors. Journal of Pediatric Psychology, 2021.

13. Nowicki, S., Manual for the receptive tests of the Diagnostic Analysis of Nonverbal Accuracy 2 (DANVA2). 2006.

14. Bonner, M.J., et al., Social functioning and facial expression recognition in survivors of pediatric brain tumors. Journal of Pediatric Psychology, 2008. 33 : p. 1142-1152.

15. Dennis, M., et al., Understanding of literal truth, ironic criticism, and deceptive praise following childhood head injury. Brain and Language, 2001. 78 : p. 1-16.

16. Dennis, M., et al., Emotional expression and socially modulated emotive communication in children with traumatic brain injury. Journal of the International Neuropsychological Society, 2013.19 : p. 34-43.

17. Gresham, F.M. and S.N. Elliott, Social Skills Improvement System - Rating Scales . 2008, Bloomington, MN: Pearson Assessments.

18. Devine, K.A., et al., PROMIS Peer Relationships Short Form: How Well Does Self-Report Correlate With Data From Peers? Journal of Pediatric Psychology, 2018. 43 (9): p. 1059-1067.

19. Hoffman, L., Longitudinal analysis: Modeling within-person fluctuation and change . 2015: Routledge.

20. Hocking, M.C., W. Hobbie, and M.J. Fisher, Development of the Pediatric Neuro-Oncology Rating of Treatment Intensity (PNORTI).Journal of neuro-oncology, 2018. 136 (1): p. 73-78.

21. Micklewright, J.L., et al., Quantifying pediatric neuro-oncology risk factors: Development of the Neurological Predictor Scale. Journal of Child Neurology, 2008. 23 : p. 455-458.

22. Willard, V.W., Social skills interventions for survivors of pediatric brain tumors: A review and reformulation. Pediatric Blood \& Cancer, 2018. e27434 .

23. Palmer, S.L., et al., Processing speed, attention, and working memory after treatment for medulloblastoma: an international, prospective, and longitudinal study. Journal of Clinical Oncology, 2013. 31 (28): p. 3494 .

24. Salley, C.G., et al., Social self-perception among pediatric brain tumor survivors compared to peers. Journal of developmental and behavioral pediatrics: JDBP, 2014. 35 (7): p. 427.

25. Hocking, M.C., et al., Face processing and social functioning in pediatric brain tumor survivors. Journal of Pediatric Psychology, 2021. 46 (10): p. 1267-1275.

\section{Hosted file}

PBC Table 1_11.23.21.docx available at https://authorea.com/users/448535/articles/547337-aprospective-study-of-social-competence-in-survivors-of-pediatric-brain-tumors

\section{Hosted file}

PBC Table 2_11.23.21.docx available at https://authorea.com/users/448535/articles/547337-aprospective-study-of-social-competence-in-survivors-of-pediatric-brain-tumors

\section{Hosted file}


PBC Table 3 11.23.21.docx available at https://authorea.com/users/448535/articles/547337-aprospective-study-of-social-competence-in-survivors-of-pediatric-brain-tumors

\section{Hosted file}

PBC Table 4 11.23.21.docx available at https://authorea.com/users/448535/articles/547337-aprospective-study-of-social-competence-in-survivors-of-pediatric-brain-tumors 